\title{
Stereotactic ablative body radiotherapy versus conventionally fractionated radiotherapy for early stage large cell neuroendocrine carcinoma of the lung
}



\author{
Rodney E Wegner*,1, Stephen Abel ${ }^{1}$ \& Athanasios Colonias ${ }^{1}$ \\ ${ }^{1}$ Division of Radiation Oncology, Allegheny Health Network Cancer Institute, Pittsburgh, PA 15212, USA \\ *Author for correspondence: Rodney.wegner@ahn.org
}

\section{Practice points}

- Some patients with early stage large cell neuroendocrine carcinoma of the lung will be ineligible for surgery.

- These patients will be managed with radiotherapy; either stereotactic ablative body radiotherapy (SABR) or conventionally fractionated radiation therapy.

- These patients are typically quite under represented on available lung SABR trials.

- This large national analysis showed that up to one third of such patients are managed with SABR.

- The median SABR dose was $50 \mathrm{~Gy}$ in four fractions and the median conventionally fractionated radiation therapy dose was 65 Gy in 33 fractions.

- Predictors of SABR included distance to facility, earlier stage, treatment at an academic facility and more recent year of treatment.

- After propensity matching patients treated with SABR had improved survival, median of 34.7 months compared with 23.7 months $(p=0.02)$.

- SABR use will likely increase for these patients and appears reasonable based on the results presented herein.

Aim: Some patients with early stage large cell neuroendocrine carcinoma (LCNEC) of the lung are not surgical candidates and will be managed with radiotherapy. We used the national cancer database to identify predictors of stereotactic radiotherapy and compare outcomes. Materials \& methods: We queried national cancer database for T1-2NO LCNEC treated with radiation. Logistic regression and Cox regression identified predictors of stereotactic ablative body radiotherapy (SABR) and survival, respectively. Results: We identified 754 patients, with 238 (32\%) treated with SABR. Predictors of SABR were distance to facility, no chemotherapy, academic center, $\mathrm{T} 1$ and recent year. After propensity matching, median survival was 34.7 months compared with 23.7 months in favor of SABR $(p=0.02)$. Conclusion: SABR for LCNEC has increased over time and was associated with improved survival.

First draft submitted: 3 February 2020; Accepted for publication: 27 March 2020; Published online: 21 April 2020

Keywords: large cell neuroendocrine $\bullet$ radiation therapy $\bullet$ SABR $\bullet$ SBRT

Large cell neuroendocrine carcinoma (LCNEC) is a rare pulmonary tumor representing $<5 \%$ of lung cancer diagnoses and having features of both non small-cell lung cancer (NSCLC) and small-cell lung cancer (SCLC). Due to its rarity, clinical management is often extrapolated from both NSCLC and SCLC guidelines [1]. As such, the gold standard treatment for early stage LCNEC is surgical resection with some data showing benefit of surgical resection over radiotherapy [2]. However, there will be a subset of patients unable to undergo surgery due to comorbidities, technical limitations, anatomic location or even patient refusal. In those situations, radiation represents an alternative treatment, with techniques ranging from daily conventionally fractionated radiation therapy (CFRT), to more hypofractionated advanced regimens.

One such technique is stereotactic body radiotherapy (SBRT), also referred to as stereotactic ablative body radiotherapy (SABR). SABR is a radiotherapeutic technique, whereby, high dose radiation is delivered in a conformal manner over a relatively small number of fractions. Having emerged over the past 15-20 years, SABR represents an 


\section{CONSORT diagram}

Stereotactic body radiation therapy (SBRT) vs conventionally fractionated radiation therapy (CFRT) in treatment of early stage large cell neuroendocrine carcinoma of the lung

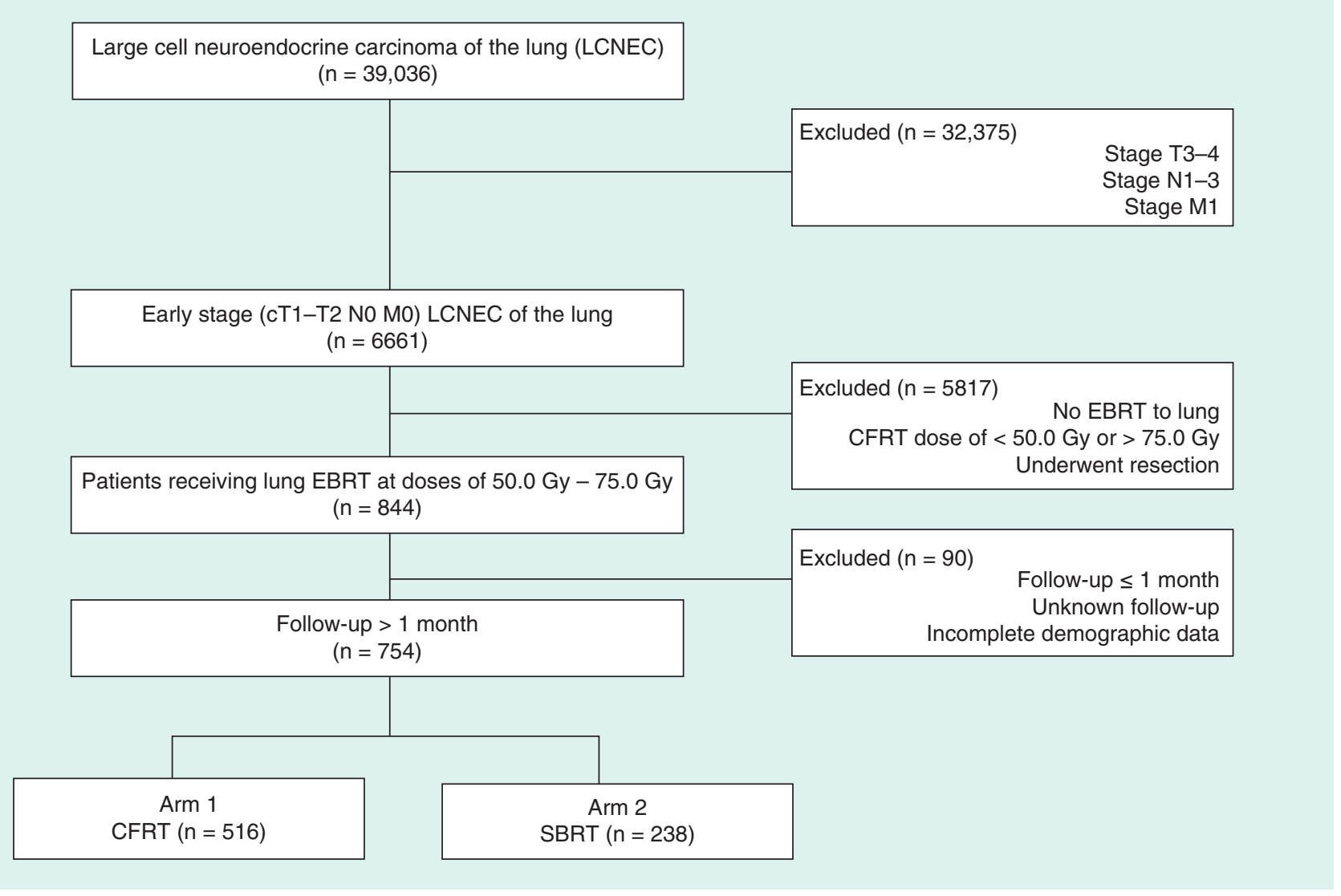

Figure 1. CONSORT diagram showing selection criteria.

excellent alternative to surgical resection for non-operative patients and is supported by high level evidence in the setting of NSCLC [3]. However, to date, nearly all studies on the subject of SABR in NSCLC have focused on more common histologies, such as squamous cell carcinoma and adenocarcinoma. Recent evidence has also suggested a potential role for SABR in treatment of early stage SCLC as well [4], however the efficacy and utilization patterns of SABR in LCNEC remains relatively unknown. Considering the paucity of evidence regarding SABR in LCNEC, we analyzed the national cancer database (NCDB) to examine trends in the use of SABR and compare outcomes in patients with LCNEC receiving SABR versus CFRT.

\section{Materials \& methods}

We queried the NCDB dataset (years: 2004-2015) for patients with NSCLC with LCNEC histology (histologic code: 8012,8013$)$ that were clinical stage T1-2N0 and treated with chest-directed radiation. Radiation technique is coded for within the NCDB and patients receiving either SBRT/SABR or definitive dose (50-75 Gy) CFRT were included. Only patients with follow-up of $\geq 1$ month from diagnosis were included to account for immortal time bias. Figure 1 is a CONSORT diagram outlining all selection criteria and patient cohorts.

The NCDB is hospital-based registry overseen and managed by the American Cancer Society and American College of Surgeons. Data provided is de-identified; thus, Internal Review Board (IRB) approval was not required 
to conduct this study. The NCDB is estimated to capture pertinent diagnostic and treatment information on approximately $70 \%$ of newly diagnosed malignancies each year across 1500 cancer centers nationwide. Of note, the American College of Surgeons and the Commission on Cancer (both IL, USA) have not verified and are not responsible for the analytic or statistical methodology employed, or the conclusions drawn from these data by the authors of this study.

Characteristics captured in the NCDB and used in this analysis included race (Caucasian, African American and other), facility type (community program, comprehensive community program and academic/research program), comorbidity score (the well regarded Charlson/Deyo score [5]) and location (metropolitan, urban and rural). Other reported socioeconomic data includes income and percentage of population with less than a high school diploma, both of which are based upon the patient's zip code.

Statistical analyses were performed with Medcalc Version 18 (Ostend, Belgium). Summary statistics are presented for discrete variables. Overall survival (OS) is recorded in months from diagnosis to death or last follow-up. Multivariable logistic regression was used to identify predictors of SABR and included all baseline characteristics. In addition, the results of the multivariable logistic regression were used to generate a propensity score indicating the likelihood of SABR based on baseline clinicopathologic characteristics [6]. Patients treated with SABR were then matched with patients treated with CFRT using an exact match on that score. This resulted in 203 matched pairs, in which a Kaplan-Meier analysis was then used to generate survival curves on those 203 pairs [7]. Finally, a multivariable Cox regression was also utilized to generate hazard ratios and predictors of worse outcome [8].

\section{Results}

We identified 754 patients meeting the eligibility criteria above, of which 238 (32\%) received SABR. Median SABR dose was 50 Gy (48-60 Gy) in four fractions (three-five fractions) corresponding to a biologically effective dose $(\mathrm{BED})_{10}>100 \mathrm{~Gy}$ which has been shown to correlate with better outcomes [9]. For patients treated with CFRT the median dose was 65 Gy (60-68 Gy) in 33 fractions (27-35 fractions). The median time from diagnosis to start of radiation was 50 days (31-78 days). The median age was 63 years old and there was a relatively even distribution of men and women. Approximately one third of patients received chemotherapy. Table 1 contains a full list of baseline patient characteristics.

On multivariable logistic regression including all baseline characteristics, predictors of SABR included: treatment at an academic facility $(p=0.001)$, distance of $>10$ miles to treatment facility $(p=0.001)$, more recent year of treatment $(\mathrm{p}<0.0001)$ and lack of chemotherapy $(\mathrm{p}<0.0001)$. Table 2 comprehensively outlines the results of that analysis. Median follow-up was 29.7 months (1.2-106.9 months). On multivariable Cox regression, predictors of worse survival were: increased age, CFRT, male sex and T2 stage (Table 3). After propensity matching (as described in the methods) 203 pairs were generated using an exact match on the propensity score. KaplanMeier analysis was done and there appeared to be a survival benefit to SABR (median survival of 34.7 vs 23.7 months; $\mathrm{p}=0.02$; Figure 2).

\section{Discussion}

Over the past decade SABR has become the standard of care for NSCLC patients ineligible for surgical resection. This technique has been studied mostly in NSCLC patients with more common histologies (i.e., adenocarcinoma and squamous cell carcinoma). As such, only a small fraction of patients treated with SABR carried a diagnosis of LCNEC (i.e., 5\% LCNEC in radiation therapy oncology group [RTOG] 0236) [3]. Not surprisingly, our analysis showed a steady increase in SABR use over time for early stage LCNEC. In addition, patients treated at academic facilities were more likely to get SABR, likely owing to expertise, availability of appropriate technology/personnel, distance or proximity and access to clinical trials.

As described above, the use of SABR skyrocketed following the initial results of RTOG 0236. In this trial, patients with medically inoperable early stage NSCLC were treated to a dose of $54 \mathrm{~Gy}$ in three fractions [3]. Local control was excellent at $>90 \%$ with minimal toxicity in patients with peripheral disease. An NCDB analysis, highlighted this shift in practice patterns demonstrating an increase in SABR use compared with CFRT in patients with early stage adenocarcinoma, squamous cell carcinoma and NSCLC not otherwise specified (NOS) [10]. For instance, in 2004 approximately $10 \%$ of patients received SABR; whereas $>90 \%$ of patients were treated with SABR in 2015. In that same study, with propensity matching there was an OS benefit to SABR (median survival of 39 vs 28 months; $\mathrm{p}<0.001$ ) compared with CFRT. Similarly, a large retrospective analysis from Memorial Sloan Kettering (NY, USA) compared outcomes in 398 patients treated with SABR (median dose 48 Gy/four fractions) 


\section{Table 1. Patient characteristics $(n=754)$.}

\begin{tabular}{|c|c|}
\hline Characteristics & Number (\%) \\
\hline \multicolumn{2}{|l|}{ Sex } \\
\hline Males & $389(52)$ \\
\hline Females & $365(48)$ \\
\hline \multicolumn{2}{|l|}{ Race } \\
\hline White & $664(88)$ \\
\hline African American & $77(10)$ \\
\hline \multicolumn{2}{|l|}{ Comorbidity score } \\
\hline 0 & $467(62)$ \\
\hline 1 & $170(22)$ \\
\hline$\geq 2$ & $117(16)$ \\
\hline \multicolumn{2}{|l|}{ Insurance } \\
\hline \multicolumn{2}{|l|}{ Education \% } \\
\hline$\geq 29$ & $156(21)$ \\
\hline $20-28.9$ & $210(28)$ \\
\hline 14-19.9 & $251(33)$ \\
\hline$<14$ & $137(18)$ \\
\hline \multicolumn{2}{|l|}{ Treatment facility type } \\
\hline Community cancer program & $65(9)$ \\
\hline Comprehensive community cancer program & $429(57)$ \\
\hline Academic/research program & $260(34)$ \\
\hline \multicolumn{2}{|l|}{ Treatment facility location } \\
\hline$>46,000$ & $162(21)$ \\
\hline \multicolumn{2}{|l|}{ Distance to treatment facility (miles) } \\
\hline $\begin{array}{l}\leq 10 \\
>10\end{array}$ & $\begin{array}{l}377(50) \\
377(50)\end{array}$ \\
\hline \multicolumn{2}{|l|}{ Age distribution (years) } \\
\hline$\leq 73$ & $399(53)$ \\
\hline$>73$ & $355(47)$ \\
\hline \multicolumn{2}{|l|}{ Grade } \\
\hline Well differentiated & $3(1)$ \\
\hline Moderately differentiated & $8(1)$ \\
\hline Poorly differentiated & $371(49)$ \\
\hline Not recorded & $372(49)$ \\
\hline \multicolumn{2}{|l|}{ Year of diagnosis } \\
\hline 2004-2006 & $237(31)$ \\
\hline 2007-2009 & $248(33)$ \\
\hline 2010-2012 & $173(23)$ \\
\hline $2013-2014$ & $96(13)$ \\
\hline
\end{tabular}




\section{Table 1. Patient characteristics $(n=754)$ (cont.).}

\begin{tabular}{|lr}
\hline Characteristics & Number (\%) \\
\hline 1 & $416(55)$ \\
\hline 2 & $338(45)$ \\
\hline Chemotherapy & $512(68)$ \\
\hline No & $242(32)$ \\
\hline Yes & \\
\hline Treatment & $238(32)$ \\
\hline SABR & $516(68)$ \\
\hline CFRT & \\
\hline $\begin{array}{l}\text { Education quantified as percentage of individuals from that zip code with less than a high school diploma. } \\
\dagger \\
\text { C Median income quantified by patient zip code. }\end{array}$ \\
\hline
\end{tabular}

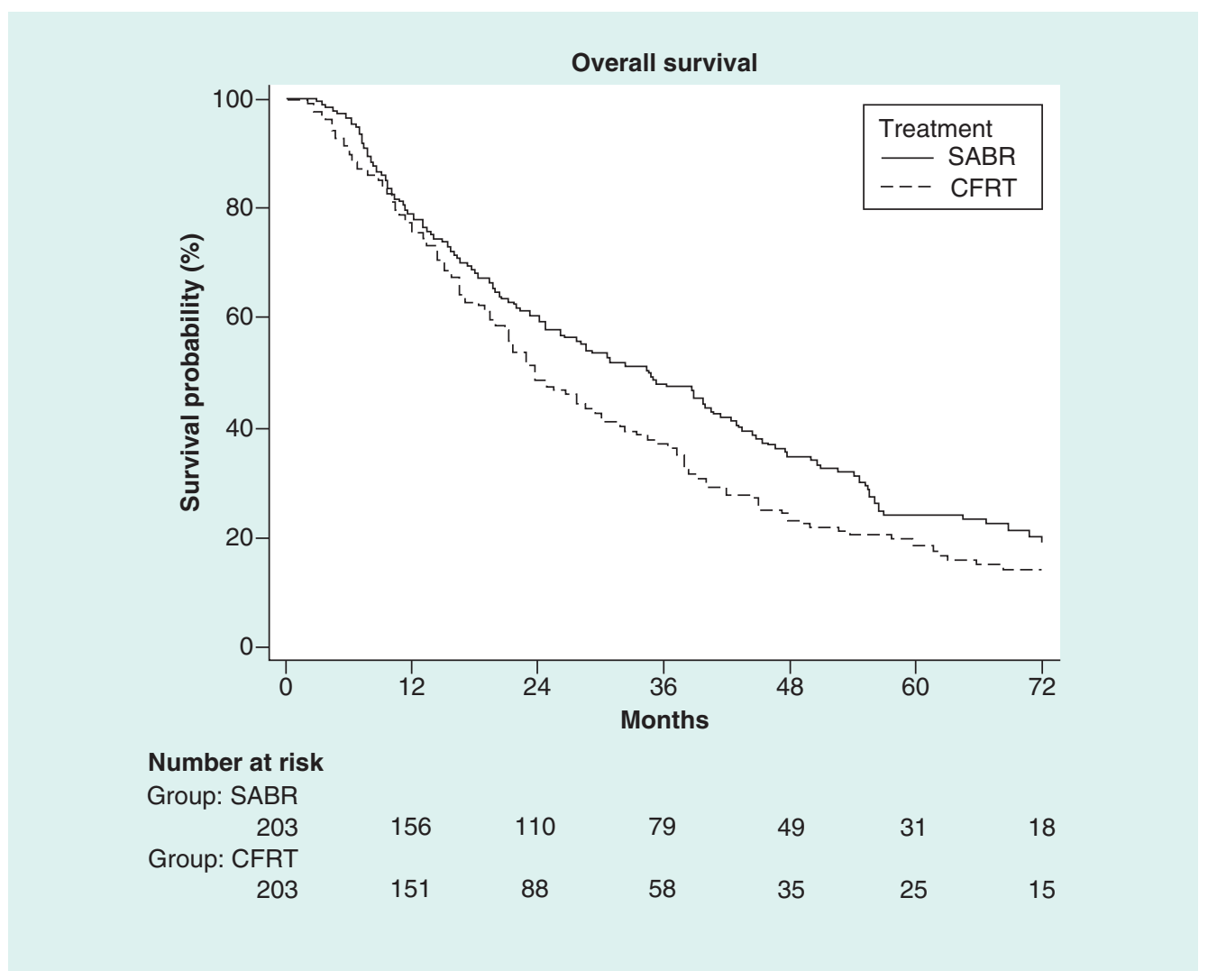

Figure 2. Propensity matched Kaplan-Meier curve showing benefit to stereotactic ablative body radiotherapy with a median overall survival 34.7 versus 23.7 months; $p=0.02$

CFRT: Conventionally fractionated radiation therapy; SABR: Stereotactic ablative body radiotherapy.

and fractionated radiation to a median dose of $75.6 \mathrm{~Gy}$ in 43 fractions [11]. Patients treated with SABR had improved local control (87 vs 66\%; $\mathrm{p}<0.001)$ and 3 year OS ( 53 vs 39\%; $\mathrm{p}=0.018)$. Most recently, a prospective randomized trial from multiple institutions in Australia and New Zealand compared SABR ( $54 \mathrm{~Gy} /$ three fractions) to CFRT (66 Gy/33 fractions). Patients were randomized in a 2:1 fashion with a primary end point of local control [12]. Both local control (89 vs 65\%; $\mathrm{p}=0.008)$ and OS (77 vs 59\%; $\mathrm{p}=0.027$ ) were improved with SABR at a median follow-up of 2 years. Importantly, $<2 \%$ of the study population (i.e., $2 / 101$ patients) were of LCNEC histology, limiting the interpretation of this study. 
Table 2. Multivariable logistic regression for stereotactic ablative body radiotherapy.

\section{Characteristics}

Sex

Males

Females

Race

White

African American

Other

Comorbidity score

0

1

$\geq 2$

Insurance

Not insured

Private payer

Government

Unrecorded

Education \%

$\geq 29$

20-28.9

14-19.9

$<14$

\section{Treatment facility type}

Community cancer program

Comprehensive community cancer program

Academic/research program

Treatment facility location

Metro

Urban

Rural

Income (\$USD ${ }^{\dagger}$ )

$<30,000$

$30,000-35,000$

$35,000-45,999$

$>46,000$

Distance to treatment facility (miles)

$\leq 10$

$>10$

Age distribution (years)

$\leq 73$

$>73$

Grade

Well differentiated

Moderately differentiated

Poorly differentiated

\section{Year of diagnosis}

2004-2006

2007-2009

2010-2012

2013-2014

* $p$ values less than or equal to 0.05 .

Education quantified as percentage of individuals from that zip code with less than a high school diploma.

$\dagger$ Median income quantified by patient zip code.

\section{Odds ratios $(95 \% \mathrm{Cl})$}

p-value

\section{Reference}

$0.83(0.56-1.24)$

0.37

Reference

$1.57(0.83-3.00) \quad 0.17$

$0.75(0.16-3.50) \quad 0.71$

Reference

$1.01(0.63-1.63) \quad 0.97$

$0.79(0.46-1.36) \quad 0.27$

Reference

$0.90(0.12-6.76) \quad 0.92$

$1.27(0.18-9.03) \quad 0.81$

$2.77(0.23-33.46) \quad 0.42$

Reference

$1.38(0.75-2.55) \quad 0.30$

$1.87(0.91-3.82) \quad 0.09$

$1.96(0.82-4.70) \quad 0.13$

Reference

$2.48(1.02-6.00) \quad 0.04$ *

$4.64(1.86-11.56) \quad 0.0010$ *

Reference

$0.72(0.40-1.33) \quad 0.30$

$1.35(0.45-4.02) \quad 0.59$

Reference

$1.27(0.69-2.35) \quad 0.44$

$1.59(0.78-3.25) \quad 0.20$

$0.92(0.40-2.11) \quad 0.84$

Reference

$2.09(1.35-3.25) \quad 0.0010^{*}$

Reference

$0.80(0.52-1.22) \quad 0.30$

\section{Reference}

$0.52(0.01-33.16) \quad 0.76$

$0.89(0.02-32.90) \quad 0.95$

Reference

$7.98(4.30-14.86)<0.0001$ *

$11.44(5.90-22.17)<0.0001$ *

$22.24(10.55-46.89) \quad<0.0001 *$ 
Table 2. Multivariable logistic regression for stereotactic ablative body radiotherapy (cont.).

\begin{tabular}{|lll|}
\hline Characteristics & Odds ratios $(95 \% \mathrm{Cl})$ & p-value \\
\hline 1 stage & & \\
\hline 1 & Reference & $<0.0001^{*}$ \\
\hline 2 & $0.41(0.27-0.62)$ & \\
\hline Chemotherapy & Reference & $<0.0001^{*}$ \\
\hline Yes & $0.15(0.09-0.26)$ & \\
\hline
\end{tabular}

* $p$ values less than or equal to 0.05 .

Education quantified as percentage of individuals from that zip code with less than a high school diploma.

$\dagger$ Median income quantified by patient zip code.

\begin{tabular}{|c|c|c|}
\hline Characteristic & Hazard ratio $(95 \% \mathrm{Cl})$ & p-value \\
\hline \multicolumn{3}{|l|}{ Age (years) } \\
\hline$\leq 74$ & Reference & \\
\hline$>74$ & $1.24(1.04-1.47)$ & 0.015 \\
\hline \multicolumn{3}{|l|}{ Education $\left(\%^{\dagger}\right)$} \\
\hline$\geq 29$ & Reference & \\
\hline 14-19.9 & $1.19(0.87-1.62)$ & 0.28 \\
\hline$<14$ & $1.29(1.05-1.59)$ & 0.016 \\
\hline \multicolumn{3}{|l|}{ Treatment } \\
\hline SBRT & Reference & \\
\hline CFRT & $1.21(1.00-1.46)$ & 0.046 \\
\hline $\mathrm{T} 1$ & Reference & \\
\hline $\mathrm{T} 2$ & $1.35(1.13-1.59)$ & 0.0005 \\
\hline
\end{tabular}

$\dagger$ Education quantified as percentage of individuals from that zip code with less than a high school diploma.

CFRT: Conventionally fractionated radiation therapy; SABR: Stereotactic ablative body radiotherapy.

SABR has also been evaluated, although not prospectively, in SCLC. Typically, SABR is not used in SCLC considering the rarity of early stage disease. Nevertheless, a multi-institutional registry study examined outcomes in 21 inoperable patients with SCLC treated to a median dose of $48 \mathrm{~Gy}$ in four fractions, with only four patients going on to systemic therapy [4]. Local control was not reported, but 1 -year survival was $73 \%$ potentially a reflection of distant spread. An NCDB study similar to the aforementioned study compared outcomes in early stage SCLC treated with SABR compared with CFRT [13]. That series included 2107 patients of which 147 were treated with SBRT (median dose 50 Gy in four fractions), while the remainder were treated with CFRT (median dose 55.8 Gy in 30 fractions). Median survival after propensity matching was 34 versus 25 months, favoring SABR. It should be noted that the National Comprehensive Cancer Network guidelines now recommend SABR for inoperable stage I SCLC [14].

As described above the data for SABR in LCNEC are rather limited, with poor or non-existent representation in most of the SABR literature. Certainly, patients with LCNEC are most likely present in several prospective studies, however, are potentially reported as NSCLC NOS [15]. This was the case in the famous SPACE trial which compared SBRT (66 Gy in three fractions) to conventional fractionation (70 Gy in 35 fractions) ultimately showing no difference in OS despite a lack of balance in prognostic factors [15]. Highlighting the paucity of available data, a review of the literature identifies only single case report which was published in 2019. This case involved a 78 year old male with early stage LCNEC who was treated with SABR at a dose of 55 Gy in five fractions [16]. He tolerated treatment well and was controlled locally at 18 months. There is another NCDB analysis examining the 
use of chemotherapy after surgical resection of early stage LCNEC [17]. That analysis showed a significant benefit to chemotherapy given after resection (hazard ratio: 0.54; $\mathrm{p}<0.0001$ ). With those results in mind, perhaps systemic therapy should be considered following SABR for inoperable patients.

There are certainly limitations that need to be discussed in regard to our study, namely its retrospective nature and inherent selection bias. Of note, patients treated with CFRT were more likely to receive chemotherapy, potentially indicating more aggressive biology or other undocumented disease characteristics which could account for the inferior outcomes. In addition, the NCDB does not document location of tumors, as more central lesions would have likely precluded SABR, especially in the earlier years of the cohort due to concerns over toxicity [18]. Moreover, the NCDB does not track toxicity data which is important when comparing these radiation techniques. There is also no documentation of local control, distant recurrence or any salvage therapy which would all be important end points/covariates to report and interpret, particularly in the setting of LCNEC which has a propensity for recurrence (both local and distant).

\section{Conclusion}

The results presented here show an increase in the use of SABR for non-operative bronchopulmonary LCNEC. Compared with CFRT, survival outcomes were superior with SABR suggesting its preferential use over CFRT. The use of SABR for early stage LCNEC will likely continue to rise and appears to be appropriate based on the data presented herein.

Author contributions

RE Wegner participated in project conception and design, statistical analysis and manuscript drafting. S Abel participated in manuscript revisions and statistical analysis. A Colonias participated in manuscript revisions.

\section{Disclaimer}

Of note, the Commission on Cancer and American College of Surgeons has not substantiated and are not responsible for the analytic or statistical methodology employed or the conclusions drawn from these data by the investigator.

Financial \& competing interests disclosure

The authors have no relevant affiliations or financial involvement with any organization or entity with a financial interest in or financial conflict with the subject matter or materials discussed in the manuscript. This includes employment, consultancies, honoraria, stock ownership or options, expert testimony, grants or patents received or pending, or royalties.

No writing assistance was utilized in the production of this manuscript.

Open access

This work is licensed under the Attribution-NonCommercial-NoDerivatives 4.0 Unported License. To view a copy of this license, visit http://creativecommons.org/licenses/by-nc-nd/4.0/

\section{References}

Papers of special note have been highlighted as: • of interest; $\bullet \bullet$ of considerable interest

1. Siegel RL, Miller KD, Jemal A. Cancer statistics, 2018. CA Cancer J. Clin. 68(1), 7-30 (2018).

2. Glisson BS, Moran CA. Large-cell neuroendocrine carcinoma: controversies in diagnosis and treatment. J. Natl Compr. Canc. Netw. 9(10), 1122-1129 (2011).

3. Timmerman R, Paulus R, Galvin J et al. Stereotactic body radiation therapy for inoperable early stage lung cancer. JAMA 303(11), 1070-1076 (2010).

- Landmark study on use of stereotactic ablative body radiotherapy in NSCLC.

4. Singh R, Ansinelli H, Sharma D et al. Clinical outcomes following stereotactic body radiation therapy (SBRT) for stage I medically inoperable small cell lung carcinoma: a multi-institutional analysis from the RSSearch patient registry. Am. J. Clin. Oncol. 42(7), 602-606 (2019).

5. Deyo RA, Cherkin DC, Ciol MA. Adapting a clinical comorbidity index for use with ICD-9-CM administrative databases. J. Clin. Epidemiol. 45(6), 613-619 (1992).

6. D'agostino RB Jr. Propensity score methods for bias reduction in the comparison of a treatment to a non-randomized control group. Stat. Med. 17(19), 2265-2281 (1998).

7. Meier ELKP. Nonparametric estimation from incomplete observations. J. Am. Stat. Assoc. 53(282), 457-481 (1958). 
8. Cox DR. Regression Models and Life- Tables. J. R. Stat. Soc. 34(2), 187-220 (1972).

9. Onishi H, Shirato H, Nagata Y et al. Hypofractionated stereotactic radiotherapy (HypoFXSRT) for stage I non-small cell lung cancer: updated results of 257 patients in a Japanese multi-institutional study. J. Thorac. Oncol. 2(7 Suppl. 3), S94-S100 (2007).

10. Haque W, Verma V, Polamraju P, Farach A, Butler EB, Teh BS. Stereotactic body radiation therapy versus conventionally fractionated radiation therapy for early stage non-small cell lung cancer. Radiother. Oncol. 129(2), 264-269 (2018).

11. Von Reibnitz D, Shaikh F, Wu AJ et al. Stereotactic body radiation therapy (SBRT) improves local control and overall survival compared to conventionally fractionated radiation for stage I non-small cell lung cancer (NSCLC). Acta Oncol. 57(11), 1567-1573 (2018).

12. Ball D, Mai GT, Vinod S et al. Stereotactic ablative radiotherapy versus standard radiotherapy in stage 1 non-small-cell lung cancer (TROG 09.02 CHISEL): a Phase III, open-label, randomized controlled trial. Lancet Oncol. 20(4), 494-503 (2019).

-• More recent randomized study showing improved outcomes for all NSCLC with use of stereotactic ablative body radiotherapy compared to conventionally fractionated radiation therapy.

13. Verma V, Hasan S, Wegner RE, Abel S, Colonias A. Stereotactic ablative radiation therapy versus conventionally fractionated radiation therapy for stage I small cell lung cancer. Radiother. Oncol. 131, 145-149 (2019).

14. Network NCC. Non Small Cell Lung Cancer V6.2019 (2019). nccn.or/professionals/physician_gls/pdf/nscl_blocks.pdf

15. Nyman J, Hallqvist A, Lund JA et al. SPACE: arandomized study of SBRT vs conventional fractionated radiotherapy in medically inoperable stage I NSCLC. Radiother. Oncol. 121(1), 1-8 (2016).

16. Jo IY, Yeo SG. Stereotactic body radiation therapy for pulmonary large cell neuroendocrine carcinoma: a case report. Onco. Targets Ther. 12, 1359-1364 (2019).

17. Kujtan L, Muthukumar V, Kennedy KF, Davis JR, Masood A, Subramanian J. The role of systemic therapy in the management of stage I large cell neuroendocrine carcinoma of the lung. J. Thorac. Oncol. 13(5), 707-714 (2018).

18. Timmerman R, Mcgarry R, Yiannoutsos C et al. Excessive toxicity when treating central tumors in a Phase II study of stereotactic body radiation therapy for medically inoperable early-stage lung cancer. J. Clin. Oncol. 24(30), 4833-4839 (2006). 